БИОРЕЗОРБИРУЕМЫЕ ВНУТРИСОСУДИСТЫЕ КАРКАСЫ. ОБЗОРНАЯ СТАТЬЯ

В.В. Плечев², И.Е. Николаева', И.В. Буваев ', И.Г. Загитов', Р.ю. Ризберг', U.E. яманаева'

1 Республиканский кардиологический центр, Россия, 450106, Уфа, ул. Степана Кувыкина, 96

${ }^{2}$ Башкирский государственный медицинский университет, Россия, 450008, Уфа, ул. Ленина, 3

Плечев Владимир Вячеславович - Д.м.Н., профессор,

зав. кафедрой госпитальной хирургии ФГБОУ ВО БГМУ

Николаева Ирина Евгеньевна - К.м.н., главный врач

Республиканского кардиологического центра

Бузаев Игорь Вячеславович - К.м.Н., зав. отделением

рентгенхирургических методов диагностики и лечения № 1

Республиканского кардиологического центра

Загитов Ильгиз Гайфуллович - врач по рентгенэндоваскулярной

диагностике и лечению, отделения РХМДИЛ № 1

Республиканского кардиологического центра

Ризберг Роман Юрьевич, - К.М.Н., врач по рентгенэндоваскуляр-

ной диагностике и лечению, отделения РХМДИЛ № 1

Республиканского кардиологического центра

Яманаева Инна Евгеньевна - К.м.Н., кардиолог отделения

РХМДИЛ № 1 Республиканского кардиологического центра

Контакты: Загитов Ильгиз Гайфуллович, e-mail: zilgiz.82@

gmail.com

Введение. Современная интервенционная хирургия - это одно из наиболее динамично развивающихся направлений современной медицины. Свидетельством этому является появление в её арсенале биорезорбируемых стентов - внутрисосудистых каркасов, обеспечивающих временную механическую поддержку и доставку цитостатических веществ в сосудистой стенке в период её регенерации. Появление данной технологии в англоязычной литературе зачастую называют четвертой революцией в рентгенохирургии. В данном литературном обзоре авторы постарались отобразить современные представления о безопасности, показаниях и недостатках биорезорбируемых каркасов.

Методы. В обзоре представлены данные 65 статей по биорезорбиуремым внутрисосудистым каркасам, найденных в сети Pubmed. Предпочтение отдавалось данным многоцентровых исследований, международных регистров, а также отчетам о клинических случаях осложнений, связанных с имплантацией биорезорбируемых внутрисосудистых каркасов.

Результат. В данном литературном обзоре представлены последние англоязычные публикации на тему применения биорезорбируемых внутрисосудистых каркасов в лечении ишемической болезни сердца, а также перечислены основные этапы их развития.

Ключевые слова: Абсорб, скафолд, биорезорбируемые внутрисосудистые каркасы, чрескожное коронарное вмешательство, стенты, стентирование, ишемическая болезнь сердца

Для цитирования: Плечев В.В., Николаева И.Е., Бузаев И.В., Загитов И.Г., Ризберг Р.Ю., Яманаева И.Е. Биорезорбируемые внутрисосудистые каркасы. Обзорная статья. Креативная хирургия и онкология. 2017;7(4):53-62. DOI:10.24060/2076-3093-2017-74-53-62.

\title{
BIORESORBABLE VASCULAR SCAFFOLDS (SYSTEMATIC LITERATURE REVIEW)
}

\author{
Vladimir V. Plechev², Irina E. Nikolaeva', Igor V. Buzaev', Ilgiz G. Zagitov', \\ Roman Yu. Rizberg', Inna E. Yamanaeva'
}

${ }^{1}$ Republic Cardiological Center, 96 Stepan Kuvykin st., Ufa, 450106, Russian Federation

${ }^{2}$ Bashkir State Medical University, 3 Lenin st., Ufa, 450008, Russian Federation

Plechev Vladimir Vyacheslavovich - Doctor of Medical Sciences,

Professor, Head of the Hospital Surgery Department in Federal State 
Budgetary Educational Institution of Higher Education Bashkir State Medical University

Nikolaeva Irina Evgenevna - Candidate of Medical Sciences, Chief Physician of the Republic Cardiological Center

Buzaev Igor Vyacheslavovich - Candidate of Medical Sciences, Head of the X-ray Surgical Methods of Diagnostics and Treatment Department №1, Republic Cardiological Center

Zagitov Ilgiz Gayfullovich - X-ray Endovascular Diagnostics and Treatment Doctor of the X-ray Surgical Methods of Diagnostics and Treatment Department №1, Republic Cardiological Center

Rizberg Roman Yurevich - Candidate of Medical Sciences, X-ray Endovascular Diagnostics and Treatment Doctor of the $X$-ray Surgical Methods of Diagnostics and Treatment Department №1, Republic Cardiological Center

Yamanaeva Inna Evgenevna - Candidate of Medical Sciences, Cardiologist of the X-ray Surgical Methods of Diagnostics and Treatment Department №1, Republic Cardiological Center Contacts: Zagitov Ilgiz, e-mail:zilgiz.82@gmail.com

Introduction: Modern interventional surgery is one of the most dynamically developing areas of modern medicine. Evidence of this is the bioresorbable stent appearance in its stockpile - intravascular frames, which provide temporary mechanical support and the delivery of cytotoxic substances in the vascular wall during its regeneration. The emergence of this technology in English literature is often called the fourth revolution in x-ray surgery. In this literature review, the authors tried to reflect modern ideas about safety, indications and disadvantages of bioresorbable scaffolds.

Methods: This review presents data of 65 articles on bioresorbable intravascular frames in pubmed. The preference was given to the data of multicenter studies and international registries and records of clinical cases of complications associated with implantation of bioresorbable intravascular scaffolds.

Results: This literature review presents the latest English-language publications on the use of bioresorbable intravascular scaffolds in the treatment of ischemic heart disease, and it lists the main stages of their development too.

Keywords: absorb, scaffold bioresorbable intravascular scaffolds, percutaneous coronary intervention, stent, stenting, absorbable stents, coronary heart disease

For citation: Plechev V.V., Nikolaeva I.E., Buzaev I.V., Zagitov I.G., Rizberg R.Yu., Yamanaeva I.E. Bioresorbable vascular scaffolds (systematic literature review). Creativesurgeryandoncology. 2017;7(4):53-62. DOI:10.24060/2076-3093-2017-7-4-53-62.

\section{ВВЕДЕНИЕ}

Появление новейших биорезорбируемых внутрисосудистых каркасов называют началом новой эры в интервенционной кардиологии, четвертой революцией в рентгенохирургии [1,2]. Однако также в свое время называли внедрение в практику цельнометаллических стентов [3], а декадой позднее - стентов с лекарственным покрытием [4]. В настоящее время выявление на коронароангиографии показаний для чрескожного коронарного вмешательства ведет к имплантации коронарного стента, в идеале покрытого цитостатиком. В результате сосуд оказывается "пожизненно заточенным в металлическую клетку» [5]. Одним из наиболее возможных сценариев дальнейшего прогрессирования заболевания является нарастание слоя эндотелия - неоинтимы - внутри стента, что происходит, несмотря на наличие цитостатического покрытия. Неоинтима в свою очередь может подвергаться дегенерации и атеросклерозу вплоть до образования атеросклеротической бляшки с дальнейшим разрывом ее в просвет стента. G. Nakazawa с соавт. опубликовали эти данные в журнале «EuroHeartJournal» за 2007 и 2011 годы [6,7]. На рисунке 1 представлено изображение, полученное при внутрисосудистом ультразвуковом исследовании передней межжелудочковой арте- рии пациентки с нестабильной стенокардией через 9 лет после имплантации металлического стента [6].

Есть мнение, что цитостатические препараты покрытых стентов нарушают метаболизм сосудистой стенки, вызывая её дегенерацию, растяжение и приводят к отсроченной приобретенной малапозиции. Это может стать причиной поздних тромбозов в стенте [1].

Существуют и более сложные биомеханические процессы в сосудистой стенке, которые нарушаются после имплантации перманентных внутрисосудистых каркасов. Так, в "Journal of Biomechanics» за 2000 год в статье «Коронарные стенты изменяют трехмерную геометрию сосуда и трехмерное напряжение сдвига» авторы указывают на то, что участки коронарных артерий, подвергнувшиеся стентированию более подвержены рестенозам в тех местах, где нормальная вазомоторная функция нарушена в большей степени [8]. В других источниках аналогичные выводы подтверждаются внутрисосудистыми ультразвуковыми исследованиями [9] и оптической когерентной томографией [10]. В указанных исследованиях авторы приходят к выводу, что после имплантации металлического стента изгиб коронарного сосуда в проксимальном участке увеличивается на $120 \%$, а в дистальном - на 100\%, что создает пред- 


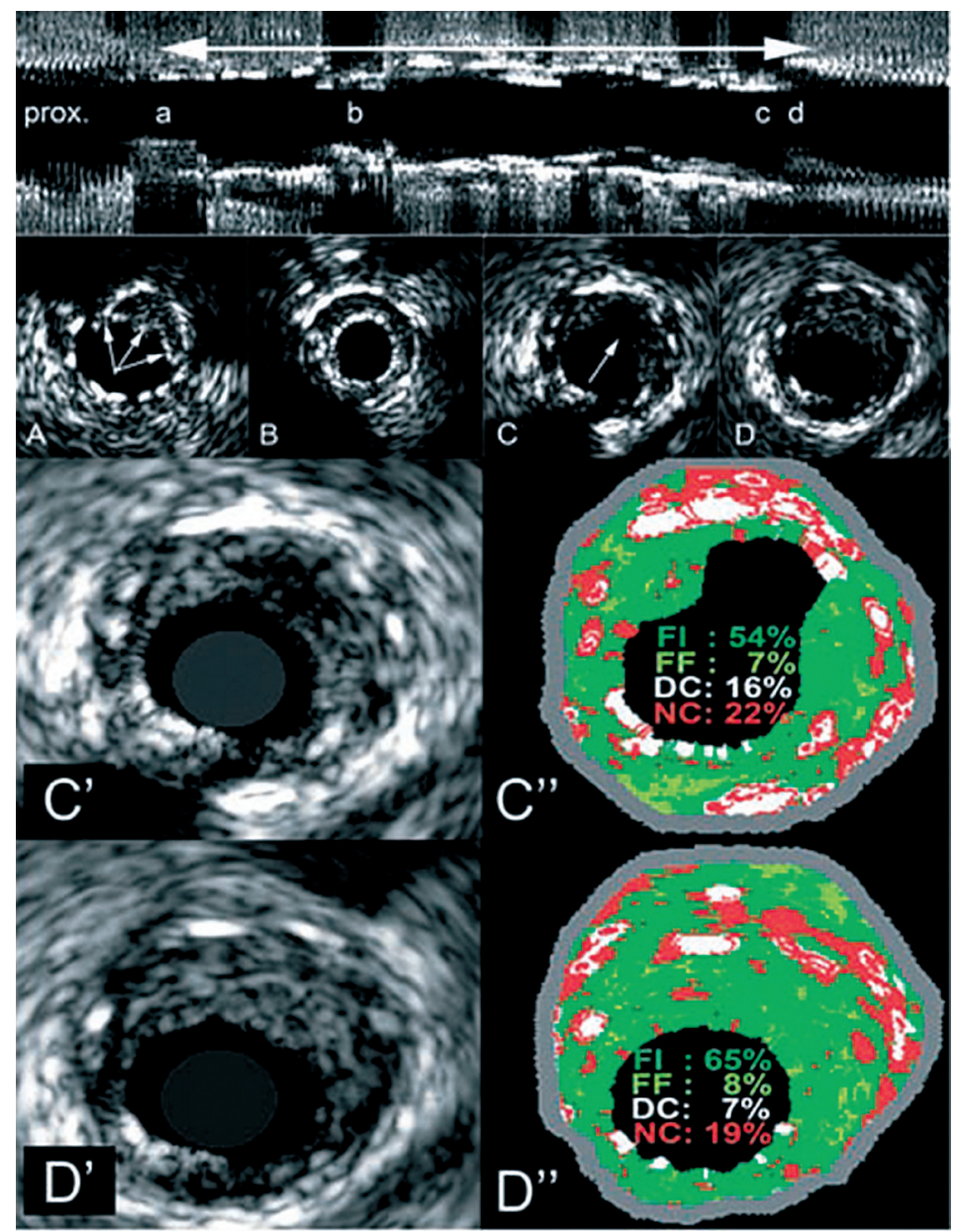

Внутрисосудистое ультразвуковое исследование. На верхнем изображении представлен продольный срез ПМЖВ, двойной стрелкой обозначен имплантированный ранее стент. Буквами от A до D обозначены поперечные срезы ПМЖВ. А - срез проксимальной ПМЖВ, стрелками обозначена малая позиция стента; В - Минимальная площадь поперечного сечения в стенте 4,6 мм2; C - Эксцентричный разрыв бляшки (обозначен стрелкой); D - Интактная бляшка внутри неоинтимы сосуда. C - Увеличенное изображение. Режим виртуальной гистологии разорванной атеросклеротической бляшки внутри неоинтимы. D - Интактная атеросклеротическая бляшка за зоной разрыва.

\section{Рисунок 1 - Внутрисосудистое ультразвуковое исследование.}

Figure 1 - Intravascular ultrasound

посылки для асимметричных рестенозов в стенте.

До сих пор остается до конца не изученным вопрос, почему стентирование ускоряет процесс неоатеросклероза. Однако достоверно известно, что при отсутствии нормальной вазомоторной функции активируются проатерогенные механизмы и ингибируются антиатерогенные. Так, в экспериментальных исследованиях с частичной лигацией сонной артерии у мышей путем выделения из тканей молекул РНК было доказано, что уменьшение подвижности сосудистой стенки ускоряет процесс атеросклероза путем активации проатерогенных генов и ингибирования антиатерогенных генов, вызывая эндотелиальную дисфункцию и воспаление [11].

С этой точки зрения выгодно отличается пластичный материал биорезорбируемых внутрисосудистых каркасов, которые создают меньше деформаций трехмерной геометрии коронарных артерий, что теоретически должно способствовать уменьшению количества рестенозов [1], а после полной резорбции каркаса - восстановлению нормальной вазомоторной функции [12]. На практике это предположение находит свое подтверждение в статье, опубликованной в журнале «ЈАСС Cardiovascular Interventions» за 2014 год, в которой авторы указывают, что несмотря на активацию проатерогенных механизмов после имплантации биодеградируемого внутрисосудистого каркаса в связи с иммобилизацией сосудистой стенки, количественно выраженной в снижении эндотелиального напряжения сдвига менее 1 Па на 62\% площади стентированного сегмента, через 2 года только 16\% площади стентированного сегмента имело величину эндотелиального напряжения сдвига менее 1 Па. Это доказывает, что после резорбции каркаса восстанавливаются нормальная геометрия и вазомоторная функция сосуда, что активирует атеропротективную активность здоровой сосудистой стенки [13].

Поэтому мечты о временном внутрисосудистом каркасе, обеспечивающем механическую поддержку сосудистой стенке после разрыва атеросклеротической бляшки баллонным катетером, о доставке цитостатического препарата и продолжительной экспозиции им в нужном месте, который исчезнет, как только в нем отпадет необходимость, не оставляли интервенционных кардиологов со времен первого установленного стента $[14,15]$, как и попытки их создания $[16,17]$. Основную идею механизма действия биорезорбируемого внутрисосудистого каркаса хорошо отображает название статьи "Biodegradable stents: they do their job and disappear = Биодеградируемые стенты: они делают свое дело и исчезают» [18].

\section{Биорезорбируемые стенты. Клинические испытания}

Первыми в клинической практике биорезорбируемые стенты из полимера молочной кислоты начали использовать японские специалисты [19]. Ими был разработан и внедрен в клиническую практику стент Igaki-Tamai® - биорезорбируемый внутрисосудистый каркас из L-хирального полимера 
молочной кислоты без лекарственного покрытия с толщиной балок 0,17 мм. Результаты 10-летнего наблюдения за пациентами были опубликованы в 2012 году в журнале «Circulation» [20]. В период с августа 1998 г. по апрель 2000 г. было прооперировано 50 пациентов с 63 стенозами коронарных артерий. Было имплантировано 84 биорезорбируемых внутрисосудистых каркасов Igaki-Tamai. За 10-летний период контакт с 2 пациентами был потерян. Зарегистрирована 1 смерть от сердечных причин, 6 несердечных смертей, 4 инфаркта миокарда. Суммарная доля повторных реваскуляризаций составила 16\% в первый год, 18\% за 5 лет, 28\% за 10 лет. Было зарегистрировано 2 доказанных случая тромбоза в стенте: 1 подострый и 1 отсроченый. В последнем случае отсроченный тромбоз был связан со стентом с лекарственным покрытием, имплантированным проксимальным каркасом Igaki-Tamai® [21].

Коронароангиографический контроль с проведением внутрисосудистого ультразвукового исследования был осуществлен через 1, 3 и 6 месяцев после имплантации. По данным внутрисосудистого ультразвукового исследования авторы указывают на то, что полная резорбция наблюдается в среднем за 3 года. Примечательно, что в журнале «ЈАСС: Cardiovascular Interventions» за 2014 год есть публикация о клинических испытаниях биорезорбируемых внутрисосудистых каркасов Igaki-Tamai для бедренной артерии с данными 12-месячного клинического наблюдения [22].

Далее последовали публикации о серии клинических испытаний биорезорбируемых внутрисосудистых каркасов Абсорб (Absorb, Abbott vascular). Материал биорезорбируемого стента Absorb состоит из L- (PLLA) (левосторонний изомер) и D- хираль ных (PDLLA) (правосторонний изомер) полимеров молочной кислоты в соотношении 1:1. Время полной резорбции PDLLA составляет 9 месяцев, а PLLA - 36 месяцев. В процессе резорбции оба этих вещества гидролизируются до молочной кислоты, которая затем утилизируется в цикле Кребса $[23,24,25]$. В качестве цитостатического препарата используется эверолимус. Согласно данным производителя примерно 80\% цитостатика выделяется в течение первых 30 дней, а остальные 20\% - за 4 месяца.

В связи с тем, что материал биорезорбируемого внутрисосудистого каркаса полимер молочной кислоты рентгенонегативен - невидим при рентгеноскопии, для точной навигации при имплантации каркаса у проксимального и дистального краев установлены титановые рентгенопозитивные метки. Кроме этого, рентгеноконтрастные титановые метки позволяют выявить место, куда был установлен биорезорбируемый внутрисосудистый каркас при контрольной коронароангиографии после того, как весь полимер каркаса резорбируется. В ходе ретроспективного анализа данных мультиспиральной компьютерной томографии, полученных в ходе исследований абсорб когорта А и когорта Б, было установлено, что через 18 месяцев после имплантации биорезорбируемых внутрисосудистых каркасов рентгеноконтрастные метки не подвержены дисло- кации в результате резорбции материала, несмотря на опасения, что в результате отрыва от резорбированного основания они могут стать причиной эмболии дистального русла [26].

\section{Absorb Cohort A [12]}

The ABSORB Cohort A - это проспективное нерандомизированное исследование без контрольной группы. Оценивалась безопасность применения биорезорбируемых внутрисосудистых каркасов Абсорб (Absorb, Abbott Vascular) в лечении ишемический болезни сердца. Первая фаза исследования была проведена в 2006 году. Тогда было прооперировано 30 пациентов. Для динамического наблюдения были доступны 29 пациентов, так как один пациент подписал отказ от дальнейшего участия в исследовании. Послеоперационное наблюдение проводилось через 1 год. 2 пациента умерли от несердечных причин: один от неходжкинской лимфомы на 888-й день, второй - от перфорации двенадцатиперстной кишки на 706-й день после имплантации биорезорбируемого внутрисосудистого каркаса. Зафиксирован 1 случай по конечной точке $(3,4 \%)$ - на 46-й день пациент поступил с клиникой острого коронарного синдрома, в анализе крови тропонины были слабо положительными. На коронарографии было зафиксировано 42\% рестенозов в биорезорбируемом внутрисосудистом каркасе. За весь период наблюдения не зарегистрировано ни одного случая тромбоза в каркасе. Через 18 месяцев произведена мультиспиральная компьютерная томография (МСКT) 25 пациентам. Оптическая когерентная томография и внутрисосудистое ультразвуковое исследование через 2 года продемонстрировали восстановление вазомоторной функции и позднее увеличение просвета сосуда. Неинвазивное исследование функционального резервного кровотока (ФРК) было произведено у 18 пациентов: дистально ФРК составил в среднем 0,86 (IQR 0,82-0,94)

\section{Absorb Cohort B}

Вторая фаза исследования (когорта Б) была начата в 2009 году и включила 101 пациента. Материал скафолда был модифицирован для увеличения радиальной жесткости. Первые 45 пациентов были запланированы для проведения коронарографии через 6 месяцев и 2 года, другие 56 - через 1 и 3 года. В первой группе краевой стеноз был зафиксирован через 6 месяцев у одного пациента. По данным внутрисосудистого ультразвукового исследования (ВСУЗИ) и оптической когерентной томографии (ОСТ) было зарегистрировано уменьшение площади скафолда на 2\%, поздняя потеря просвета на

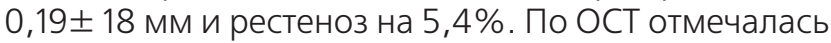
эндотелизация в 96,8\% случаев. Малая позиция хотя бы одной страты, первоначально отмеченная у 12 пациентов, на контроле была подтверждена только у 3 [27]. Во второй группе отмечены уменьшение площади на 16,8\% по ВСУЗИ и на 20\% по ОСТ, поздняя потеря просвета 0,27士0,32мм и стеноз 1,94\% (ВСУЗИ). Эндотелизация в 96,69\% случаев. Малая 
позиция хотя бы одной страты 12, на контроле 4 [1]. Из 101 пациента у 3 отмечалось повышение сердечных маркеров и у 3 была проведена повторная реваскуляризация $(5,9 \%$ конечная точка) $[27,28]$.

\section{The Absorb Extend study}

В след за когортой А и Б в настоящее время проводится исследование Абсорб Экстенд. Это мультицентровое нерандомизированное исследование без наличия контрольной группы. В него запланировано включить 800 пациентов из 100 клиник. Из них 50 пациентов запланированы для установки 2-х стентов внахлест, 100 пациентов - для проведения МCKT контроля. Эти две субгруппы по плану будут анализироваться отдельно. В настоящее время есть предварительные результаты исследования первых 512 пациентов. Согласно нему, было 8 случаев инфарктов миокарда без образования Q-зубца в течение первых 7 дней (NQMI), а также 3 случая QMI в течении 30 дней. Суммарно 11 (2,1\%) пациентов по конечной точке за первые 30 дней. 2,9\% и 4,3\% за 180 и 365 дней соответственно [25].

Absorb II - это мультицентровое, рандомизированное исследование, сравнивающее Absorb второй генерации с XIENCE - эверолимусом, содержащим металлический стент. Всего для участия в исследовании запланирован 501 пациент с соотношением Absorb:XIENCE 2:1. Послеоперационное наблюдение запланировано на 30- и 180-й дни и через 1, 2 и 3 года.

Absorb III - это крупное проспективное рандомизированное мультицентровое исследование, произведенное в США с целью получения разрешения на клиническое применение биодеградируемых внутрисосудистых каркасов Абсорб [29,30]. 2008 пациентов со стабильной стенокардией были случайным образом разделены на 2 группы в соотношении 2:1. Пациентам первой группы были имплантированы биорезорбируемые внутрисосудистые каркасы Абсорб (Absorb, Abott Vascular), пациентам второй группы имплантирован покрытый эверолимусом стент Xience. За первичную конечную точку была принята комбинация из сердечных смертей инфаркта миокарда с поражением стентированной артерии и повторной реваскуляризации, вызванной ишемией. Группы пациентов были однородные. Единственным процедурным отличием являлась более частая постдилатация в группе Абсорб (65,5\% против 51,2\%, p<0,001). Согласно выводам

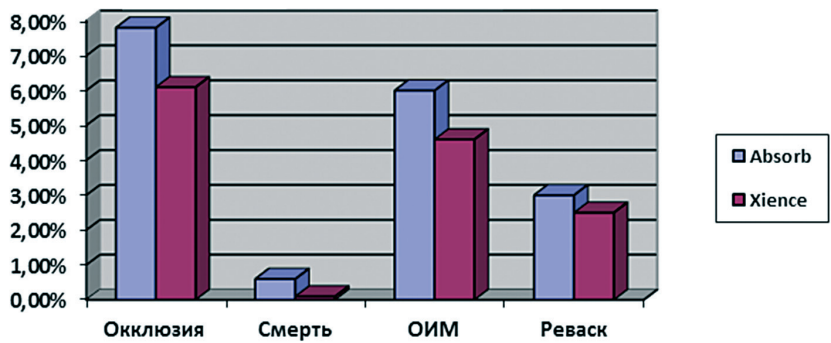

Рисунок 2 - Индивидуальные компоненты конечной точки исследования Absorb III.

Figure 2 - The individual components of the Absorb III endpoint study. по результатам исследования, Absorb оказался не хуже, чем Xience, частота конечной точки составила соответственно 7,8\% и 6,1\%, доверительный интервал - 0,5-3,9; ( $p=0,007)$. Результаты по индивидуальным компонентам отображены на рисунке 2.

\section{ABSORB Japan [31]}

ABSORB Japan - это мультицентровое, слепое, рандомизорованное, проспективное исследование, проведенное в Японии для получения допуска к использованию Abosrb в этой стране. Проводилось сравнение стентов Absorb (Abbott Vascular) с кобальтохромовым эверолимус пропитанным стентом (название стента в исследовании не указанно, обозначается CoCrEES). Рандомизация проводилась в соотношении 2:1. Всего включено 400 пациентов, из них 266 пациентам были имплантированы стенты Absorb, 134 - CoCrEES. В качестве первичной конечной точки была взята совокупность сердечных смертей, инфарктов миокарда и повторных реваскуляризаций в том же сегменте, вызванных ишемией. Вторичной конечной точкой была принята поздняя потеря просвета при ангиографическом контроле через 13 месяцев. Через 12 месяцев в группе биорезорбируемых каркасов наблюдалось 4,2\% исходов по первичной конечной точке против 3,8\% в группе CoCrEES. Поздняя потеря просвета через 13 месяцев при ангиографическом контроле составила 0,13 \pm 0,30 мм в группе биорезорбируемых каркасов и 0,12 0 0,32 мм в группе CoCrEES. На рисунке 3 представлены индивидуальные компоненты конечной точки.

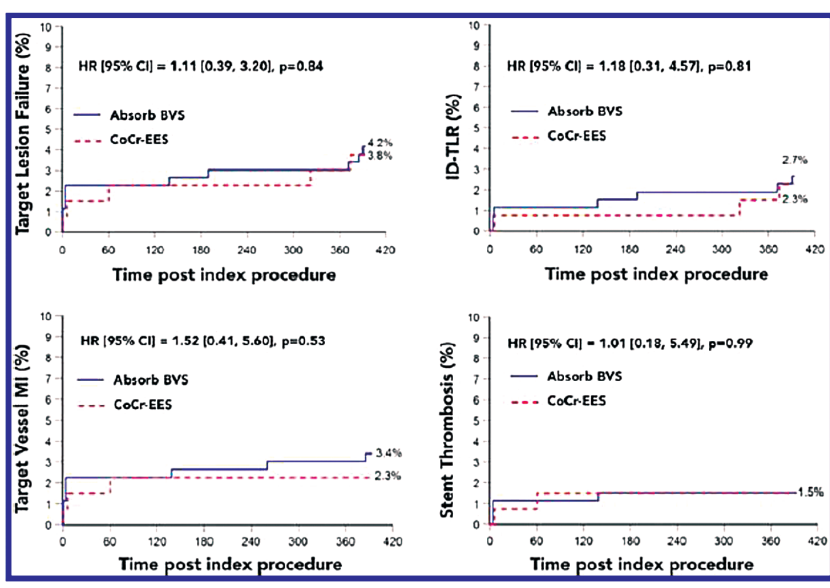

Рисунок 3 - Absorb Japan.

Figure 3 - Absorb Japan.

\section{Absorb China [29]}

Проспективное рандомизированное клиническое исследование было проведено в Китае для получения в этой стране разрешения применения биорезорбируемых внутрисосудистых каркасов. 480 пациентов были рандомизированы на две группы: экспериментальная и контрольная в соотношении 1:1. В экспериментальной группе были имплантированы биорезорбируемые внутрисосудистые каркасы Абсорб, а в контрольной - стенты Xience V. В качестве конечной точки было принято считать 
уменьшение площади поперечного сечения более чем на 0,15 мм при коронароангиографическом контроле через 1 год после имплантации биорезорбируемого внутрисосудистого каркаса. По результатам исследования уменьшение площади просве-

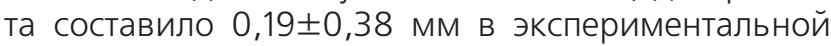

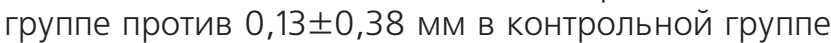
$(p=0,01)$. В ходе исследования не было зарегистрировано ни одного случая тромбоза в течение года наблюдения.

The AMC Single Centre Real World PCI Registry это проспективное клиническое исследование без контрольной группы, проведенное в Академическом медицинском центре Амстердама в период с 2012 по 2013 годы. Суммарно было прооперировано 135 пациентов, из которых 54 (39\%) с острым коронарным синдромом (из них 13\% с острым инфарктом миокарда с подъемом сегмента ST). Всего было имплантировано 159 биорезорбируемых внутрисосудистых каркасов, из которых 102 в сложных участках (типа В2 и С). Количественная коронароангиография выявила средний прирост просвета на

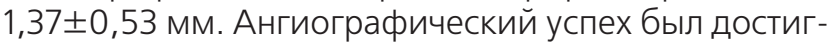
нут в 152 случаях (96\%). 97\% пациентов поступили в клинику повторно через 6 месяцев для контрольного исследования. Результат по конечной точке наблюдался у 8,5\%, из которых в 3,0\% случаев был зафиксирован инфаркт миокарда, в 3,0\% тромбозов в биорезорбируемом внутрисосудистом каркасе, 6,3\% - повторная реваскуляризация, вызванная возвратом стенокардии.

Italian Diffuse/Multivessel Disease ABSORB Prospective Registry (IT-DISAPPEARS) [32] - это проспективное мультицентровое исследование без контрольной группы, начатое в 2015 году в Италии с целью изучения безопасности биорезорбируемых внутрисосудистых каркасов Абсорб при лечении сложных поражений. Проводится в 50 клинических институтах Италии. Критерии включения: пациенты со стабильной и прогрессирующей стенокардией, с многососудистыми или протяженными (с длиной поражения более 24 мм) поражениями коронарных артерий. Клинический контроль запланирован через 5 лет. В качестве первичной конечной точки принято считать суммарную частоту сердечной смерти, нефатального инфаркта миокарда, связанного со стентированным сосудом и повторной реваскуляризацией в том же сегменте, вызванной ишемией [32].

\section{Биорезорбируемые каркасы и концепция регенеративной хирургии}

Данные исследований, указанных выше, позволяют сделать следующие выводы:

1. Биорезорбируемые каркасы обеспечивают достаточную поддержку сосудистой стенке после проведения чрескожного коронарного вмешательства.

2. Процесс биодеградации приводит к ослаблению хронического воспалительного процесса в сосудистой стенке.

3. Биорезорбируемые внутрисосудистые каркасы позволяют проводить повторные эндоваску- лярные и хирургические реваскуляризации в случае рестенозов.

4. Биорезорбируемые внутрисосудистые каркасы не создают помех для неинвазивных исследований (МСКТ и МРТ) сосудов сердца.

Интересные данные приводятся в исследовании Абсорб когорты А [17]. Через 2 года после имплантации биорезорбируемых внутрисосудистых каркасов в подгруппе пациентов проведено исследование вазомоторной функции. 9-ти пациентам проведена проба с ацетилхолином, вазодилатация более 3\% отмечена у 5 пациентов. После введения нитроглицерина получено значительное расширение сегмента с биорезорбируемым внутрисосудистым каркасом. 7-ми пациентам проведена проба с метигрелином - выявлена значительная вазоконстрикция сегмента с раннее имплантированным биорезорбируемым внутрисосудистым каркасом. Эти результаты подтверждаются аналогичными пробами у пациентов из исследования Абсорб когорты Б на 12-й месяц [28]. В сентябрьском выпуске журнала «Eurointervention» за 2016 год приводятся результаты исследования пациентов из исследования Абсорб когорты А через 5 лет после имплантации биорезорбируемых внутрисосудистых каркасов. Аналогично с исследованием [12] пациентам проводились внутрисосудистое исследование с помощью ультразвука, оптическая когерентная томография, построение виртуальной гистологии и тесты на вазореактивность [33]. 8-ми из 16 пациентов когорты проведена коронароангиография с внутрисосудистой визуализацией. Было выявлено увеличение просвета по сравнению с аналогичным двухлетним контролем, 2,14 $\pm 0,38$ мм против 1,95 $\pm 0,37$ мм; $p=0,09$ [54]. Данные внутрисосудистого ультразвукового исследования показали увеличение максимальной площади просвета $(6,96 \pm 1,13$ мм2) по сравнению с шестимесячным увеличением $(6,17 \pm 0,74$ мм2; $p=0,06)$ и двухлетним $(6,56 \pm 1,16 \mathrm{~mm} 2 ; p=0.12)$, в основном благодаря стабильному уменьшению площади атеросклеротической бляшки на протяже-

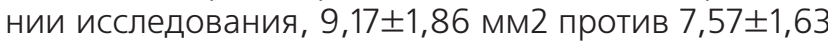
мM2; $p=0,03)$ [33].

Изложенные данные говорят о восстановлении функции эндотелия после имплантации биорезорбируемого внутрисосудистого каркаса, что позволяет отнести метод лечения рассасывающимися внутрисосудистыми каркасами к принципиально другой категории в отличие от традиционных чрескожных коронарных вмешательств с металлическими стентами.

\section{Биорезорбируемые внутрисосудистые каркасы. Недостатки}

Несмотря на все вышесказанное, биорезорбируемые внутрисосудистые каркасы обладают рядом потенциальных недостатков. Наиболее критичными из них, по нашему мнению, являются слабая радиальная жесткость балок каркаса из биорезорбируемого материала, а также хрупкость (недостаточная эластичность) полимера молочной кислоты по сравнению с кобальтохромовым сплавом тради- 
ционных стентов, вызывающая перелом и миграцию страт стента с последующим риском тромбоза $[34,35,36]$.

По результатами исследования Absorb Cohort A trial [37] выявили значимое острое и позднее (через 6 месяцев) эластическое спадение просвета сегмента, на основании чего был сделан вывод о недостаточной радиальной жесткости биорезорбируемого внутрисосудистого каркаса. С целью увеличения радиальной жесткости, а также для улучшения растяжимости без риска перелома страт производители пошли по пути утолщения балок каркаса. В результате полученный Absorb стали называть версией 1.1 [30]. Несмотря на это, производители категорически не рекомендуют перерастягивать биорезорбируемый внутрисосудистый каркас больше чем на 0,5 мм от номинального размера в связи с большой вероятностью перелома балок каркаса.

Толщина балок каркаса Абсорб 1.1 составляет 0,15 мм (для сравнения толщина страт стента Xience 0,081 мм, а биорезорбируемого внутрисосудистого каркаса Igaki-Tamai - 0,17 мм). Первые металлические стенты имели толщину, сопоставимую с толщиной современных биорезорбируемых каркасов [38]. Теоретически большая толщина страт каркаса Абсорб делает его более тромбогенным. Так, известно, что стенты с толщиной страты 0,162 мм в 1,5 раза чаще подвержены тромбозам, чем стенты со стратами толщиной 0,081 мм ( $p<0,001)$ [39]. Однако есть экспериментальные исследования in vitro, которые доказывают, что страты абсорб не являются более тромбогенными [38]. Возможно, это связано с тем, что цитостатическое покрытие биорезорбируемого внутрисосудистого каркаса уменьшает риск тромбозов [39]. С другой стороны, большая толщина балок каркасов приводит к альтерации эндотелиального сдвига напряжения - увеличению напряжения сдвига в верхней точке балок и уменьшению напряжения сдвига в нижней точке, что может провоцировать агрегацию тромбоцитов [40]. Существуют опасения, что участками наибольшей тромбогенной угрозы могут стать области перехлеста двух соседних биорезорбируемых каркасов, так как суммарная толщина балок будет составлять уже 0,3 мм [38]. Более того, экспериментальные исследования на свиньях показали, что восстановление неоинтимального слоя в таких участках задерживается на 30 дней, что эквивалентно примерно 90 дням отсроченного восстановления эндотелиального слоя у человека [41]. Вероятно это и послужило причиной того, что производители категорически не рекомендуют имплантировать каркасы Абсорб внахлест [42].

Проблема механической хрупкости при перерастяжении особенно актуальна из-за рентгенонегативных свойств биорезорбируемых каркасов, в результате чего деформация каркаса может оказаться в свое время не выявленной на рентгене при имплантации [2]. Также, вовремя не диагностированной может оказаться недостаточное раскрытие биорезорбируемого каркаса, приводящее к малой позиции - диастазу между сосудистой стенкой и балками каркаса, что создает предпосылки для острого тромбоза [43]. Так в журнале «Circulation» за 2011 год авторы статьи указывают, что стенты с малой позицией в 1,58 раза чаще $(p=0,001)$ подвержены тромбозам, а стенты, имплантированные внахлест, в 2,32 раза чаще $(p<0,001)$ [39]. Вместе с тем материал биорезорбируемого внутрисосудистого каркаса является проницаемым для света, что создает идеальные условия для исследования с помощью оптической когерентной томографии [44].

\section{Способы улучшения клинических результа- тов имплантации биорезорбируемых внутри- сосудистых каркасов}

В связи с растущим количеством имплантируемых биорезорбируемых каркасов возникают вопросы о выборе оптимальной тактики и установки [45].

Известно, что недораскрытие стентов, малпозиция страт, пролапс тканей через ячейки стентов, приводят к увеличению случаев тромбозов и рестенозов в стенте $[46,47,48,49]$. Использование внутрисосудистых методов визуализации, в частности внутрисосудистого ультразвукового метода, в качестве контроля при имплантации традиционных цельнометаллических и покрытых стентов, позволяет уменьшить количество рестенозов и число повторных реваскуляризаций $[50,51,52,53,54,55]$. Появившийся позднее метод оптической когерентной томографии, благодаря высокой разрешающей способности, позволяет с большей точностью выявлять и оценивать такие явления, как краевая диссекция, тромбоз в стенте, пролапс тканей, перелом и малпозиция страт $[56,57,58]$. Точность и разрешающая способность оптической когерентной томографии настольно велики, что существует понятие виртуальной гистологии $[44,59,60,61,62]$. Также было показано, что использование оптической когерентной томографии при стентировании традиционными стентами улучшает клинический исход $[45,63]$. Использование же метода оптической когерентной томографии в качестве контрольного исследования влияет в 40\% случаев на интраоперационную тактику [64]. Последнее утверждение нашло свое подтверждение в последующих публикациях $[62,65]$.

Информация о конфликте интересов. Конфликт интересов отсутствует.

Информация о спонсорстве. Данная работа не финансировалась.

\section{СПИСОК ЛИТЕРАTУРЫ/REFERENCES}

1. Serruys P.W, Garcia-Garcia H.M, Onuma Y. From metallic cages to transient bioresorbable scaffolds: Change in paradigm of coronary revascularization in the upcoming decade? Eur Heart J. 2012;33 (1):16-25. DOI: 10.1093/eurheartj/ehr384.

2. Ormiston J.A, Serruys P.W.S. Bioabsorbable coronary stents. Circ Cardiovasc Interv. 2009;2(3): 25560. DOI:10.1161/CIRCINTERVENTIONS.109.859173.

3. Sigwart U., Puel J., Mirkovitch V., Joffre F., Kappenberger L. Intravascular stents to prevent occlusion and restenosis after transluminal angioplasty. 
N Engl J Med. 1987:316(12):701-6. DOI: 10.1056/ NEJM198703193161201.

4. Rensing B.J, Vos J., Smits P.C, Foley D.P, Van Den Brand M.J.B.M, Van Der Giessen W.J., et al. Coronary restenosis elimination with a sirolimus eluting stent: First European human experience with 6-month angiographic and intravascular ultrasonic followup. Eur Heart J. 2001;22(22):2125-30. DOI: 10.1053/ euhj.2001.2892

5. Radu M.D. The Clinical Atlas of Intravascular Optical Coherence Tomography (OCT) for iPad. Eur Heart J. 2012;33(10):1174-5. DOI: 10.1093/eurheartj/ ehs102.

6. Ramcharitar S., Garcia-Garcia H.M. Nakazawa G., Kukreja N., Ligthart J., Virmani R., et al. Ultrasonic and pathological evidence of a neo-intimal plaque rupture in patients with bare metal stents. Eurolntervention. 2007;3(2):290-1. PMID: 19758954.

7. Nakazawa G., Otsuka F., Nakano M., Vorpahl M., Yazdani S.K., Ladich E., et al. The pathology of neoatherosclerosis in human coronary implants baremetal and drug-eluting stents. J Am Coll Cardiol. 2011;57(11):1314-22. DOI: 10.1016/j.jacc.2011.01.011.

8. Wentzel J.J., Whelan D.M., van der Giessen W.J., van Beusekom H.M.M., Andhyiswara I., Serruys P.W., et al. Coronary stent implantation changes 3-D vessel geometry and 3-D shear stress distribution. J Biomech. 2000;33(10):1287-95. PMID: 10899339.

9. Samady H., Eshtehardi P., McDaniel M.C., Suo J., Dhawan S.S., Maynard C., et al. Coronary artery wall shear stress is associated with progression and transformation of atherosclerotic plaque and arterial remodeling in patients with coronary artery disease. Circulation. 2011;124(7):779-88. DOI:10.1161/ CIRCULATIONAHA.111.021824.

10. Gy ngy si M., Yang P., Khorsand A., Glogar D. Longitudinal straightening effect of stents is an additional predictor for major adverse cardiac events. J Am Coll Cardiol. 2000;35(6):1580-9. DOI:. org/10.1016/S0735-1097(00)00570-2.

11. Nam D., Ni C.-W., Rezvan A., Suo J., Budzyn K., Llanos A., et al. Partial carotid ligation is a model of acutely induced disturbed flow, leading to rapid endothelial dysfunction and atherosclerosis. Am J Physiol Heart Circ Physiol. 2009;297(4):1535-43. DOI: 10.1152/ajpheart.00510.2009.

12. Dudek D., Onuma Y., Ormiston J.A., Thuesen L., Miquel-Hebert K., Serruys P.W. Four-year clinical follow-up of the ABSORB everolimus-eluting bioresorbable vascular scaffold in patients with de novo coronary artery disease: The ABSORB trial. Eurolntervention. 2012;7(9):1060-1. DOI: 10.4244/ EIJV7I9A168.

13. Bourantas C.V., Papafaklis M.I., Garcia-Garcia H.M., Farooq V., Diletti R., Muramatsu T., et al. Shortand long-term implications of a bioresorbable vascular scaffold implantation on the local endothelial shear stress patterns. JACC Cardiovasc Interv. 2014;7(1):1001. DOI: 10.1016/j.jcin.2013.01.139.

14. Waksman R. Biodegradable stents: they do their job and disappear. J Invasive Cardiol. 2006;18(2):70-4. PMID: 16446520.
15. Stack R.S,, Califf R.M,, Phillips H.R,, Pryor D.B,, Quigley P.J,, Bauman R.P., et al. Interventional cardiac catheterization at Duke Medical Center. Am J Cardiol. 1988;62(10, Pt 2):3F-24F. PMID: 2972185.

16. van der Giessen W.J., Slager C.J., van Beusekom H.M., van Ingen Schenau D.S., Huijts R.A., Schuurbiers J.C., et al. Development of a polymer endovascular prosthesis and its implantation in porcine arteries. J Interv Cardiol. 1992;5(3):175-85. PMID: 10150957.

17. van der Giessen W.J., Lincoff A.M., Schwartz R.S., van Beusekom H.M., Serruys P.W., Holmes D.R., et al. Marked inflammatory sequelae to implantation of biodegradable and nonbiodegradable polymers in porcine coronary arteries. Circulation. 1996;94(7):16907. PMID: 8840862.

18. Waksman R. Biodegradable stents: they do their job and disappear. J Invasive Cardiol. 2006;18(2):70-4. PMID: 16446520

19. Tamai H., Igaki K., Kyo E., Kosuga K., Kawashima A., Matsui S., et al. Initial and 6-month results of biodegradable poly-l-lactic acid coronary stents in humans. Circulation. 2000;102(4):399-404 DOI:10.1161/01.CIR.102.4.399.

20. Nishio S., Kosuga K., Igaki K., Okada M., Kyo E., Tsuji T., et al. Long-term (>10 Years) clinical outcomes of first-in-human biodegradable polyI-lactic acid coronary stents: Igaki-Tamai stents. Circulation. 2012;125(19):2343-52. DOI: 10.1161/ CIRCULATIONAHA.110.000901.

21. Cook S., Ladich E., Nakazawa G., Eshtehardi P., Neidhart M., Vogel R., et al. Correlation of intravascular ultrasound findings with histopathological analysis of thrombus aspirates in patients with very late drugeluting stent thrombosis. Circulation. 2009;120(5):3919. DOI: 10.1161/CIRCULATIONAHA.109.854398.

22. Werner M., Micari A., Cioppa A., Vadal G., Schmidt A., Sievert H., et al. Evaluation of the biodegradable peripheral Igaki-Tamai stent in the treatment of de novo lesions in the superficial femoral artery: The GAIA study. JACC Cardiovasc Interv. 2014;7(3):305-12. DOI: 10.1016/j.jcin.2013.09.009.

23. Hildick-Smith D., Lassen J.F., Koo B.K. One or two stents for coronary bifurcation lesions? Eurolntervention. 2010;6(Suppl J(8):J61-4. DOI:10.1016/S0735-1097(00)00534-9.

24. Oberhauser J.P., Hossainy S., Rapoza R.J. Design principles and performance of bioresorbable polymeric vascular scaffolds. Eurolntervention. 2009;5(Suppl F):F15-22. DOI: 10.4244/EIJV5IFA3.

25. Abizaid A., Ribamar Costa J., Bartorelli A.L., Whitbourn R., van Geuns R.J., Chevalier B., et al. The ABSORB EXTEND study: preliminary report of the twelve-month clinical outcomes in the first 512 patients enrolled. Eurolntervention. 2015;10(12):1396-401. DOI: 10.4244/EIJV10I12A243.

26. Jin Jang W., Hoon Yang J., Choi S.-H., Bin Song Y., Hahn J.-Y., Choi J.-H., et al. Fate of bioresorbable vascular scaffold metallic radio-opaque markers at the site of implantation after bioresorption. J Am Coll Cardiol Intv. 2015;8(8):271-9. DOI: 10.1016/j.jcin.2015.04.010.

27. Serruys P.W., Onuma Y., Ormiston J.A., De Bruyne B., Regar E., Dudek D., et al. Evaluation of 
the second generation of a bioresorbable everolimus drug-eluting vascular scaffold for treatment of de novo coronary artery stenosis: Six-month clinical and imaging outcomes. Circulation. 2010; 122(22):2301-12. DOI: 10.1161/CIRCULATIONAHA.110.970772.

28. Serruys P.W., Onuma Y., Dudek D., Smits P.C., Koolen J., Chevalier B., et al. Evaluation of the second generation of a bioresorbable everolimuseluting vascular scaffold for the treatment of de Novo Coronary Artery stenosis: 12-month clinical and imaging outcomes. J Am Coll Cardiol. 2011;58(15):1578-88. DOI: 10.1016/j.jacc.2011.05.050

29. Gogas B.D., King S.B., Samady H. Bioresorbable polymeric scaffolds for coronary revascularization: Lessons learnt from ABSORB III, ABSORB China, and ABSORB Japan. Glob Cardiol Sci Pract. 2015;2015(5):62. DOI: $10.5339 /$ gcsp.2015.62.

30. Kereiakes D.J., Ellis S.G., Popma J.J., Fitzgerald P.J., Samady H., Jones-McMeans J., et al. Evaluation of a fully bioresorbable vascular scaffold in patients with coronary artery disease: Design of and rationale for the ABSORB III randomized trial. Am Heart J. 2015;170(4):641-651. DOI: 10.1016/j.ahj.2015.07.013.

31. Kimura T., Kozuma K., Tanabe K., Nakamura S., Yamane M., Muramatsu T., et al. A randomized trial evaluating everolimus-eluting Absorb bioresorbable scaffolds vs. everolimus-eluting metallic stents in patients with coronary artery disease: ABSORB Japan. Eur Heart J. 2015;36(47):3332-42. DOI: 10.1093/ eurheartj/ehv435.

32. Testa L., Biondi Zoccai G., Tomai F., Ribichini F., Indolfi C., Tamburino C., et al. Italian Diffuse/ Multivessel Disease ABSORB Prospective Registry (ITDISAPPEARS). Study design and rationale. J CardiovasC Med (Hagerstown). 2015;16(3):253-8. DOI: 10.2459/ JCM.0000000000000219.

33. Simsek C., Karanasos A., Magro M., GarciaGarcia H.M., Onuma Y., Regar E., et al. Longterm invasive follow-up of the everolimus-eluting bioresorbable vascular scaffold: five-year results of multiple invasive imaging modalities. Eurolntervention. 2016;11(9):996-1003. DOI: 10.4244/EIJY14M10_12.

34. Ruiz-Salmer n R.J., Pereira S., de Araujo D. Bioresorbablevascularscaffold collapsecauses subacute thrombosis. J Invasive Cardiol. 2014;26(7):E98-9. PMID: 24993999.

35. Miyazaki T., Panoulas V.F., Sato K., Naganuma T., Latib A., Colombo A. Acute stent thrombosis of a bioresorbable vascular scaffold implanted for STsegment elevation myocardial infarction. Int J Cardiol. 2014;174(2):e72-74. DOI: 10.1016/j.ijcard.2014.04.108.

36. Mart n-Reyes R., Jim nez-Valero S., Navarro F., Moreno R. Subacute drug-eluting stent thrombosis caused by stent underexpansion: evaluation by optical coherence tomography. Case Rep Med. 2011;2011:1-3. DOI: $10.1155 / 2011 / 129341$

37. Serruys P.W., Ormiston J.A., Onuma Y., Regar E., Gonzalo N., Garcia-Garcia H.M., et al. A bioabsorbable everolimus-eluting coronary stent system (ABSORB): 2-year outcomes and results from multiple imaging methods. Lancet. 2009;373(9667):897-910. DOI: 10.1016/S0140-6736(09)60325-1.
38. Ishibashi Y., Onuma Y., Muramatsu T., Nakatani S., Iqbal J., Garcia-Garcia H.M., et al. Lessons learned from acute and late scaffold failures in the ABSORB EXTEND trial. Eurolntervention. 2014;1-9. DOI: 10.4244 /EIJV1014A78.

39. Kolandaivelu K., Swaminathan R., Gibson W.J., Kolachalama V.B., Nguyen-Ehrenreich K.L., Giddings V.L., et al. Stent thrombogenicity early in high-risk interventional settings is driven by stent design and deployment and protected by polymer-drug coatings. Circulation.2011;123(13):1400-9. DOI: 10.1161/ CIRCULATIONAHA.110.003210.

40. Papafaklis M.I., Bourantas C.V., Farooq V., Diletti R., Muramatsu T., Zhang Y., et al. In vivo assessment of the three-dimensional haemodynamic micro-environment following drug-eluting bioresorbable vascular scaffold implantation in a human coronary artery: Fusion of frequency domain optical coherence tomography and angiography. Eurolntervention. 2013;9(7):890. DOI: 10.4244/ EIJV9I7A147.

41. Farooq V., Serruys P.W., Heo J.H., Gogas B.D., Onuma Y., Perkins L.E., et al. Intracoronary optical coherence tomography and histology of overlapping everolimus-eluting bioresorbable vascular scaffolds in a porcine coronary artery model: the potential implications for clinical practice. JACC Cardiovasc Interv. 2013;6(5):523-32. DOI: 10.1016/j.jcin.2012.12.131.

42. Rzeszutko L., Depukat R., Dudek D. Biodegradable vascular scaffold ABSORB BVSTM - scientific evidence and methods of implantation. Adv Interv Cardiol. 2013;9(1):22-30. DOI:10.5114/ pwki.2013.34026.

43. Uren N.G., Schwarzacher S.P., Metz J.A., Lee D.P., Honda Y., Yeung A.C., et al. Predictors and outcomes of stent thrombosis: An intravascular ultrasound registry. Eur Heart J. 2002;23(2):124-32. DOI: 10.1053/euhj.2001.2707.

44. Mattesini A., Pighi M., Konstantinidis N., Ghione M., Kilic D., Foin N., et al. Optical coherence tomography in bioabsorbable stents: mechanism of vascular response and guidance of stent implantation. Minerva Cardioangiol. 2014;62(1):71-82. PMID: 24500218.

45. Gomez-Lara J., Diletti R., Brugaletta S., Onuma Y., Faroog V., Thuesen L., et al. Angiographic maximal luminal diameter and appropriate deployment of the everolimus-eluting bioresorbable vascular scaffold as assessed by optical coherence tomography: An ABSORB cohort $B$ trial sub-study. Eurolntervention. 2012;8(2):214-24. DOI: 10.4244/EIJV8I2A35.

46. Nakamura S., Colombo A., Gaglione A., Almagor Y., Goldberg S.L., Maiello L., et.al. Intracoronary ultrasound observations during stent implantation. Circulation. 1994;89(5):2026-34. PMID: 8181126.

47. Ong D.S., Jang I.-K. Causes, assessment, and treatment of stent thrombosis-intravascular imaging insights. Nat Rev Cardiol. 2015;12(6):325-36. DOl: 10.1038/nrcardio.2015.32.

48. Uren N.G., Schwarzacher S.P., Metz J.A., Lee D.P., Honda Y., Yeung A.C., et al. Predictors 
and outcomes of stent thrombosis: an intravascular ultrasound registry. Eur Heart J. 2002;23(2):124-32. DOI: 10.1053/euhj.2001.2707.

49. Cheneau E., Leborgne L., Mintz G.S., Kotani J., Pichard A.D., Satler L.F., et al. Predictors of subacute stent thrombosis: results of a systematic intravascular ultrasound study. Circulation. 2003;108(1):43-7. DOI: 10.1161/01.CIR.0000078636.71728.40.

50. Fitzgerald P.J., Oshima A., Hayase M., Metz J.A., Bailey S.R., Baim D.S., et al. Final results of the Can Routine Ultrasound Influence Stent Expansion (CRUISE) study. Circulation. 2000;102(5):523-30. DOI:10.1161/01.CIR.102.5.523Circulation.

51. Casella G., Klauss V., Ottani F., Siebert U., Sangiorgio P., Bracchetti D. Impact of intravascular ultrasound-guided stenting on long-term clinical outcome: a meta-analysis of available studies comparing intravascular ultrasound-guided and angiographically guided stenting. Catheter Cardiovasc Interv. 2003;59(3):314-21. DOI: 10.1002/ccd.10537.

52. Oemrawsingh P.V., Mintz G.S., Schalij M.J., Zwinderman A.H., Jukema J.W., van der Wall E.E. Intravascular ultrasound guidance improves angiographic and clinical outcome of stent implantation for long coronary artery stenoses: final results of a randomized comparison with angiographic guidance (TULIP Study). Circulation. 2003;107(1):62-7. PMID: 12515744.

53. Costa M.A., Angiolillo D.J., Tannenbaum M., Driesman M., Chu A., Patterson J., et al. Impact of stent deployment procedural factors on long-term effectiveness and safety of sirolimus-eluting stents ( final results of the multicenter prospective STLLR trial). Am J Cardiol. 2008;101(12):1704-11. DOl:10.1016/j. amjcard.2008.02.053.

54. Cheneau E., Leborgne L., Mintz G.S., Kotani J., Pichard A.D., Satler L.F., et al. Predictors of subacute stent thrombosis: results of a systematic intravascular ultrasound study. Circulation. 2003;108(1):43-7. DOI: 10.1161/01.CIR.0000078636.71728.40.

55. de Jaegere P., Mudra H., Figulla H., Almagor Y., Doucet S., Penn I., et al. Intravascular ultrasoundguided optimized stent deployment. Immediate and 6 months clinical and angiographic results from the Multicenter Ultrasound Stenting in Coronaries Study (MUSIC Study). Eur Heart J. 1998;19(8):1214-23. PMID: 9740343.

56. Takarada S., Imanishi T., Liu Y., Ikejima H., Tsujioka H., Kuroi A., et al. Advantage of nextgeneration frequency-domain optical coherence tomography compared with conventional time-domain system in the assessment of coronary lesion. Catheter Cardiovasc Interv. 2010;75(2):202-6. DOI: 10.1002/ ccd.22273.
57. Fujino Y., Bezerra H.G., Attizzani G.F., Wang W., Yamamoto H., Chami D., et al. Frequency-domain optical coherence tomography assessment of unprotected left main coronary artery disease-a comparison with intravascular ultrasound. Catheter Cardiovasc Interv. 2013;82(3):E173-83. DOI: 10.1002/ccd.24843.

58. Fujino Y., Attizzani G.F., Bezerra H.G., Wang W., Tahara S., Yamamoto H., et al. Serial assessment of vessel interactions after drug-eluting stent implantation in unprotected distal left main coronary artery disease using frequency-domain optical coherence tomography. JACC Cardiovasc Interv. 2013;6(10):103545. DOI: $10.1016 / j . j c i n .2013 .05 .015$.

59. Regar E., Schaar J.A., Mont E., Virmani R., Serruys P.W.. Optical coherence tomography. Cardiovasc Radiat Med. 2003;4(4):198-204. DOl: 10.1161/CIRCULATIONAHA.109.921528.

60. Finn A.V. Illuminating culprit plaque histology by optical coherence tomography: shedding new light on old insights. JACC Cardiovasc Interv. 2015;8(9):117779. DOI: $10.1016 /$ j.jcin.2015.05.002.

61. Gambichler T., Pljakic A., Schmitz L. Recent advances in clinical application of optical coherence tomography of human skin. Clin Cosmet Investig Dermatol. 2015;8:345-54. DOI: 10.2147/CCID.S69119.

62. Gogas B.D., Radu M., Onuma Y., Perkins L., Powers J.C., Gomez-Lara J., et al. Evaluation with in vivo optical coherence tomography and histology of the vascular effects of the everolimus-eluting bioresorbable vascular scaffold at two years following implantation in a healthy porcine coronary artery model: implications of pilot resu. Int J Cardiovasc Imaging. 2012;28(3):499-511. DOI: 10.1007/s10554011-9860-z.

63. Prati F., Di Vito L., Biondi-Zoccai G., Occhipinti M., La Manna A., Tamburino C., et al. Angiography alone versus angiography plus optical coherence tomography to guide decision-making during percutaneous coronary intervention: the Centro per la Lotta contro I'InfartoOptimisation of Percutaneous Coronary Intervention (CLI-OPCI) study. Eurolntervention. 2012;8(7):823-9. DOI: 10.4244 /EIJV8I7A125.

64. Allahwala U.K., Cockburn J.A., Shaw E., Figtree G.A., Hansen P.S., Bhindi R. Clinical utility of optical coherence tomography (OCT) in the optimisation of Absorb bioresorbable vascular scaffold deployment during percutaneous coronary intervention. Eurolntervention. 2015;10(10):1154-9. DOI: 10.4244/ EIJV10I10A190.

65. Asad A.A., Reddy K., Agarwala M.K., Dikshit B., Rath P.C., Purohit B.V., et al. Optical coherence tomography (OCT) guided deployment of ABSORB biovascular scaffolds in percutaneous coronary interventions. Indian Heart J. 2013;65:S89-90. DOl: 10.4244/EIJV10I10A190. 